\title{
Comunicação
}

\section{Farinhas de soja sem lipoxigenase agregam valor sensorial em bolos}

\author{
Maria Inês de Souza Dantas ${ }^{1}$, Mariana Gonçalves da Silva ${ }^{2}$, Cássia Regina Rezende Pinto ${ }^{1}$, Conceição Angelina \\ dos Santos Pereira ${ }^{1}$, Valéria Paula Rodrigues Minim³, Maria Christina Barros Bittencourt ${ }^{4}$
}

\section{RESUMO}

Este trabalho teve como objetivo verificar a viabilidade de se adicionar a farinha de soja à de trigo na elaboração de bolos, quantificando a aceitação dos produtos por meio de testes sensoriais afetivos, análise de componentes principais e mapa de preferência interno. Foram avaliadas quatro amostras de bolo com substituição da farinha de trigo por concentrações de 45 e $75 \%$ de farinha de soja elaborada com diferentes variedades de soja (convencional e sem lipoxigenase). Os bolos foram avaliados por consumidores, empregando-se uma escala hedônica estruturada de nove pontos. Todas as amostras obtiveram notas acima do ponto de corte $(6,0)$. As amostras elaboradas com farinha de soja de sabor melhorado foram as mais aceitas. Os resultados mostraram que a utilização da farinha de soja em substituição parcial à farinha de trigo é uma alternativa viável, constituindo em fonte agregadora de qualidade nutricional na elaboração de alimentos.

Palavras-chave: Aceitação, mapa de preferência interno, bolo de soja.

\section{ABSTRACT}

\section{Soy Flours without lipoxygenase improve sensory value in cakes}

This work aimed to evaluate the viability of adding soybean flour to wheat flour for cake making by quantifying product acceptance using affective sensorial tests, principal analysis components and internal preference map. Four cake samples with wheat flour replaced by concentrations of $45 \%$ and $75 \%$ of soybean flour made of different soybean varieties (conventional and without lipoxygenase) were evaluated. The cakes were evaluated by consumers using a structured hedonic scale of 9 points. All samples obtained grades above the cutoff point (6.0). The samples made of improved flavor soybean flour were the best accepted. The results showed that the use of soybean flour as a partial substitute for wheat flour is a viable alternative, constituting an aggregating source of nutritional quality in food making.

Key words: Acceptance, internal preference map, soybean cake.

Recebido para publicação em junho de 2006 e aprovado em fevereiro de 2010

'Nutricionista, Doutra. Universidade Federal de Viçosa, Departamento de Nutrição e Saúde, Av. P. H. Rolfs, s/n, Campus Universitário, 36570-000, Viçosa, MG. msdantas@ufv.br, casantos@ufv.br

${ }^{2}$ Nutricionista. Universidade Federal de Viçosa, Departamento de Nutrição e Saúde, Av. P. H. Rolfs, s/n, Campus Universitário, $36570-000$ Viçosa, MG. mariana_nut2003@yahoo.com.br

${ }_{3}^{3}$ Engenheira de Alimentos, Doutora. Universidade Federal de Viçosa, Departamento de Tecnologia de Alimentos, Av. P. H. Rolfs, s/n, Campus Universitário, 36570-000, Viçosa, MG.vprm@ufv.br

${ }^{4}$ Economista doméstica. Universidade Federal de Viçosa, Departamento de Tecnologia de Alimentos, Av. P. H. Rolfs, s/n, Campus Universitário, 36570-000, Viçosa, MG 


\section{INTRODUÇÃO}

A soja é uma importante alternativa alimentar na nutrição humana, devido principalmente ao alto teor e qualidade proteica (Monteiro et al., 2004). Contém lipídios com alta concentração de ácidos graxos polinsaturados, teores consideráveis de vitaminas do complexo $\mathrm{B}$, fibras alimentares, oligossacarídeos com potencial prebiótico, como rafinose e estaquiose, vitaminas e minerais (Fuchs, 2005). Além dessas vantagens, apresenta também benefícios à saúde humana para a prevenção e tratamento de doenças cardiovasculares, câncer, osteoporose e sintomas da menopausa (Hasler, 1998; Penha et al., 2007).

Entretanto, o uso de soja como ingrediente na obtenção de produtos alimentícios tem sido limitado pelo seu sabor de “feijão cru”. O Brasil, mesmo sendo o segundo maior produtor de soja do mundo, destina apenas 3\% da sua produção à alimentação humana, a maior parte é exportada e usada para ração animal (Kinouchi et al., 2002).

O sabor característico, conhecido como Beany Flavour, é atribuído à presença das lipoxigenases, enzimas que oxidam os ácidos graxos poli-insaturados existentes no grão, em especial o ácido linoleico (Morais \& Silva, 2000; Morais et al., 2000; Tsukamoto et al., 2001). Programas de melhoramento genético têm sido utilizados para produção de variedades de soja mais adequadas ao consumo humano. Entre os objetivos desses programas estão a melhora do sabor, o aumento do teor de proteína, a redução de fatores antinutricionais e melhora do aspectos físico do grão (Carrão-Panizzi, 2000).

Outra alternativa para otimizar o consumo humano de soja é a elaboração de produtos derivados. A farinha de soja é o seu produto menos refinado, porém é o industrializado mais importante, pois é usada para enriquecer alimentos e para obtenção de texturizados, concentrados e isolados. Segundo El-Dash et al. (1994), a incorporação de soja ao pão aumenta não só a qualidade em termos nutricionais, mas também a quantidade de proteína. A adição de apenas $12 \%$ de farinha de soja desengordurada, com teor médio de $52 \%$ de proteína, à farinha de trigo eleva o conteúdo protéico de 11 para 16\%, significando aumento de quase $50 \%$.

A determinação da aceitação pelo consumidor é parte crucial no desenvolvimento de um produto e envolve uma série de procedimentos. Para isso, pode-se dispor de métodos afetivos, que medem as atitudes subjetivas de aceitação ou preferência de um produto. Quando os dados da aceitação são analisados por técnicas estatísticas univariadas, assume-se que o critério de aceitabilidade dos consumidores seja homogêneo, o que implica que os valores obtidos dessa forma podem não refletir a média real. Por essa razão, a variabilidade individual dos dados também deve ser considerada, e a estrutura dos dados analisada. Tais análises podem ser realizadas pelo método estatístico denominado Mapa de Preferência Interno. A técnica desse Mapa utiliza análise estatística multivariada para obter, num espaço multidimensional, uma re- presentação gráfica das diferenças de aceitação entre produtos, identificando o indivíduo e suas preferências. Permite, portanto, identificar as amostras mais aceitas pela maioria da população do estudo (MINIM, 2006).

O objetivo do presente trabalho foi avaliar a aceitabilidade de bolos elaborados com diferentes variedades e concentrações de farinhas de soja, utilizando-se a técnica de mapa de preferência interno.

\section{MATERIAL E MÉTODOS}

\section{Elaboração da farinha de soja}

Foram utilizadas duas linhagens de soja. Uma variedade comercial e a variedade de soja UFVTN 105, desenvolvida pelo Programa de Melhoramento da Qualidade da Soja do Bioagro (Instituto de Biotecnologia Aplicado à Agricultura) da Universidade Federal de Viçosa (UFVMG), sem as enzimas lipoxigenases LOX1, LOX2 e LOX3, denominadas triplas nulas (TN).

Para a elaboração das farinhas integrais, os grãos de soja foram selecionados e submetidos ao processo de limpeza, por meio de lavagem rápida com água e secos à temperatura ambiente. A seguir foram submetidos à temperatura de $150{ }^{\circ} \mathrm{C}$, por 30 minutos, em estufa com circulação de ar (marca Marconi). Posteriormente, os grãos foram triturados em liquidificador da marca WALITA e passados em peneira de $840 \mu \mathrm{m}$ (20 mesh).

\section{Formulação dos bolos}

As formulações foram desenvolvidas por modificações da formulação padrão de bolo básico, conforme apresentação da Tabela 1.

A massa foi processada em batedeira planetária ARNO. Inicialmente o açúcar, óleo de soja e as gemas de ovos foram misturados por 10 minutos, em velocidade média. A seguir, adicionaram-se a farinha de trigo e farinha de soja, alternando com leite, e misturou-se em massa a velocidade média por cinco minutos. Posteriormente, adicionaram-se o fermento químico e a clara em neve, sem utilização da batedeira. A massa foi colocada em formas retangulares previamente untadas com óleo e farinha de trigo e assada em forno convencional por 40 minutos, a $180^{\circ} \mathrm{C}$.

\section{Teste de aceitação}

A aceitação das quatro formulações de bolo de soja foi avaliada com a participação de 89 consumidores potenciais. Os testes foram realizados em dois supermercados da cidade de Viçosa-MG.

As amostras foram apresentadas aos consumidores em blocos casualizados, de forma monádica sequencial. Os provadores avaliaram a aceitação global das formulações utilizando escala hedônica estruturada de nove pontos (Figura 1). 
Para obtenção do Mapa de Preferência Interno ou Análise de Preferência Multidimensional (MDPREF), os dados de aceitação (teste de consumidor) foram organizados numa matriz de amostras (em linhas) e consumidores (em colunas), e essa submetida à Análise de Componentes Principais (ACP) (MINIM, 2006).

Tabela 1. Formulações de bolos elaborados com farinha mista de trigo e soja

\begin{tabular}{lcccc}
\hline \multirow{2}{*}{ Ingredientes (g) } & \multicolumn{4}{c}{ Formulações } \\
\cline { 2 - 5 } & $\mathbf{C}_{\mathbf{4 5}}{ }^{*}$ & $\mathbf{C}_{\mathbf{7 5}}{ }^{*}$ & $\mathbf{M}_{\mathbf{4 5}}{ }^{* *}$ & $\mathbf{M}_{\mathbf{7 5}}{ }^{* *}$ \\
\hline Farinha de trigo & 156,75 & 71,25 & 156,75 & 71,25 \\
Farinha de soja & 128,25 & 213,75 & 128,25 & 213,75 \\
Óleo de soja & 40 & 40 & 40 & 40 \\
Ovos & 138 & 138 & 138 & 138 \\
Açúcar & 205 & 205 & 205 & 205 \\
Fermento químico & 10 & 10 & 10 & 10 \\
Leite integral & 170 & 170 & 170 & 170 \\
\hline
\end{tabular}

${ }^{*} \mathrm{C}_{45}$ e $\mathrm{C}_{75}$. Formulações contendo farinha proveniente da soja comercial, na concentração de 45 e 75\%, respectivamente.

** $\mathrm{M}_{45}$ e $\mathrm{M}_{75}$ : Formulações contendo farinha proveniente da soja melhorada, variedade UFVTN 105, na concentração de 45 e $75 \%$, respectivamente.
Nome Data
Por favor, avalie a amostra utilizando a escala abaixo para descrever o quanto você gostou ou desgostou do produto. Marque a posição da escala que melhor reflita seu julgamento
Código da amostra
( ) Gostei extremamente
( ) Gostei muito
( ) Gostei moderadamente
( ) Gostei ligeiramente
( ) Indiferente
( ) Desgostei ligeiramente
( ) Desgostei moderadamente
( ) Desgostei muito
( ) Desgostei extremamente

Figura 1. Modelo da ficha empregada no teste de consumidor utilizando escala hedônica
Os resultados foram expressos em um gráfico de dispersão das amostras (tratamentos) em relação aos dois primeiros componentes principais e às loadings (cargas) da ACP (correlações dos dados de cada consumidor com os dois primeiros componentes principais).

\section{RESULTADOS E DISCUSSÃO}

Os resultados da avaliação da aceitabilidade sensorial das quatro amostras de bolo de soja estão apresentados na Tabela 2. Observou-se que as quatro amostras obtiveram boa aceitação, com nota média acima de 6,5. As formulações contendo farinha de soja da variedade UFVTN 105, de sabor melhorado, apresentaram melhor aceitação em relação à variedade comercial. A substituição de 75\% de farinha de trigo por farinha de soja de sabor melhorado não teve rejeição entre o grupo de 89 consumidores entrevistados, enquanto a amostra de mesma concentração de farinha de soja comercial apresentou $18 \%$ de rejeição no mesmo grupo avaliado. Esse fato pode estar associado à rejeição ao sabor característico de soja apresentado pela variedade comercial, o que implica dizer que a isenção de lipoxigenases da soja pode interferir positivamente na aceitação de produtos elaborados com soja.

Com os dados obtidos no teste de aceitação das quatro amostras de bolo de soja foi realizada a análise do Mapa de Preferência Interno (Figura 2). O primeiro componente principal (PC) explicou $68 \%$ e o segundo $25 \%$, totalizando, portanto, 93\% da variância entre as amostras quanto a sua aceitação.

Na Figura 2, cada ponto representa as correlações entre os dados de aceitação de um consumidor e os dois primeiros componentes principais. Os consumidores correlacionados com pelo menos um dos componentes consideram diferença na aceitação das amostras. Para a grande maioria, houve correlação positiva com o primeiro componente principal, indicando que atribuíram notas mais elevadas para as amostras de bolo elaboradas com farinha de soja sem lipoxigenase (amostras mais à direita no gráfico, Figura 2).

Tabela 2. Nota média de aceitação e percentagem de aprovação, indiferença e rejeição de bolos de soja elaborados com diferentes variedades e concentrações

\begin{tabular}{lcccc}
\hline \multirow{2}{*}{ Amostra } & Nota Média & \multicolumn{2}{c}{ Porcentagem } \\
\cline { 3 - 5 } & & Aceitação & Indiferença & Rejeição \\
\hline C45\% & $6,9^{\mathrm{a}}$ & 82,15 & 7,87 & 3,37 \\
$\mathrm{C} 75 \%$ & $6,7^{\mathrm{a}}$ & 79,44 & 5,62 & 17,99 \\
M45\% & $7,4^{\mathrm{b}}$ & 93,26 & 3,37 & 0,00 \\
M75\% & $7,5^{\mathrm{b}}$ & 96,63 & & \\
\hline
\end{tabular}

${ }^{*} \mathrm{C}_{45}$ e $\mathrm{C}_{75}$ F Formulações contendo farinha proveniente da soja comercial, na concentração de 45 e $75 \%$, respectivamente.

$* * \mathrm{M}_{45}$ e $\mathrm{M}_{75}$ :Formulações contendo farinha proveniente da soja melhorada, variedade UFVTN 105, na concentração de 45 e $75 \%$, respectivamente.

*** Médias seguidas por letras iguais não diferem entre si ( $>$ > 0,05) pelo teste de Tukey. 


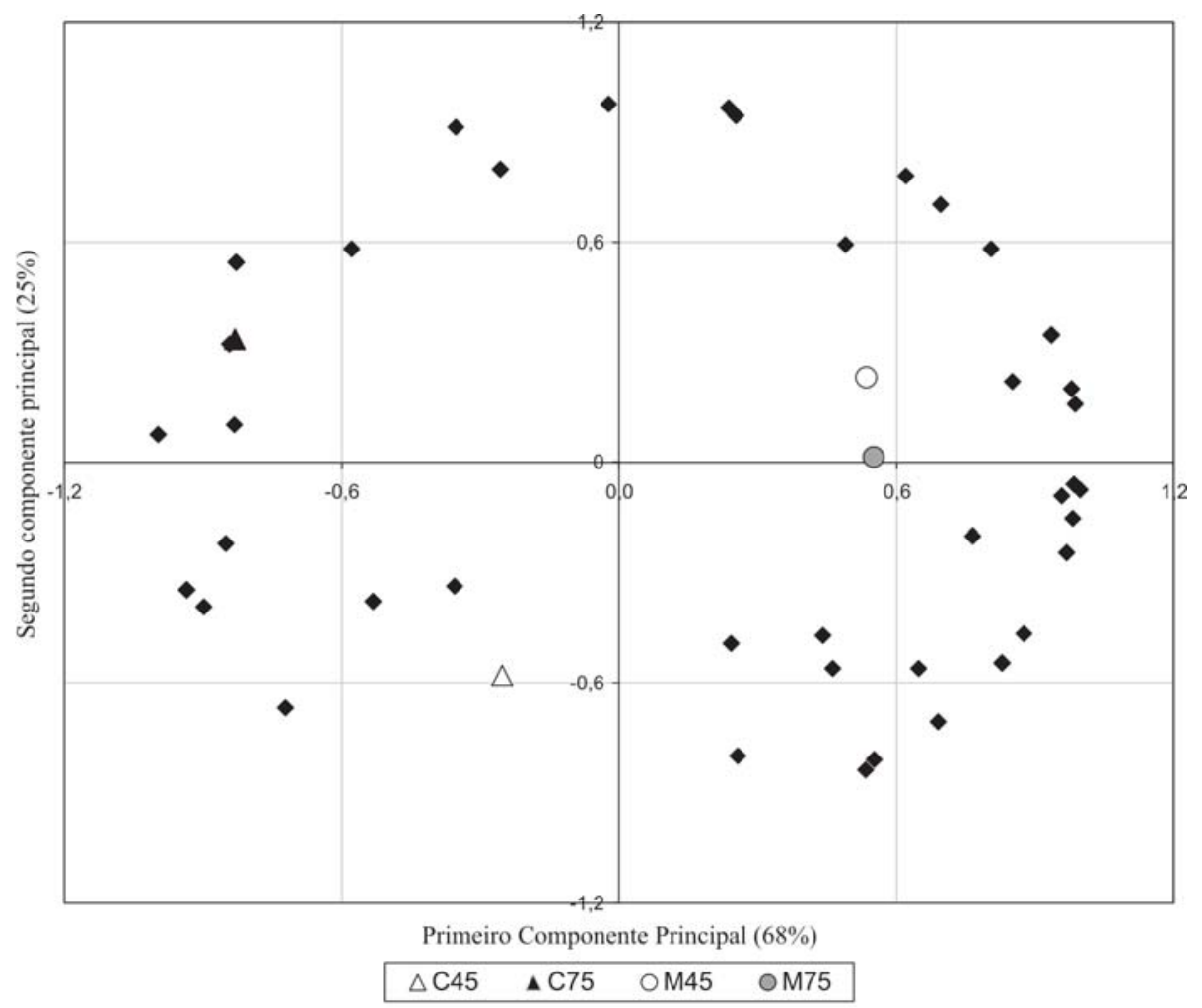

Figura 2. Mapa de preferência interno de bolo de soja

$\mathrm{C}_{45}$ e $\mathrm{C}_{75}$ : Formulações contendo farinha proveniente da soja comercial, na concentração de 45 e $75 \%$, respectivamente.

$\mathrm{M}_{45}$ e $\mathrm{M}_{75}$ Formulações contendo farinha proveniente da soja melhorada variedade UFVTN 105, na concentração de 45 e $75 \%$, respectivamente.

\section{CONCLUSÕES}

A boa aceitação de bolos elaborados com a substituição parcial da farinha de trigo por farinha de soja demonstra a viabilidade da produção de bolos agregando a proteína proveniente da soja, contribuindo para a otimização do consumo humano dessa leguminosa.

A melhor aceitação das formulações constituídas pela farinha de soja de sabor melhorado sugere que a utilização dessas em produtos alimentícios pode ser a solução para o viés da rejeição dos produtos de soja devido ao sabor residual característico da soja convencional.

\section{AGRADECIMENTOS}

Ao Programa de Melhoramento da Qualidade da Soja do Bioagro (Instituto de Biotecnologia Aplicado à Agricultura) da Universidade Federal de Viçosa (UFV-MG), pela doação da soja UFVTN 105.

\section{REFERÊNCIA}

Carrão-Panizzi MC (2000) Melhoramento genético da soja para a obtenção de cultivares mais adequados ao consumo humano. Revista Brasileira de Nutrição Clinica, 15:330-340.
El-Dash A, Mazzari MR \& Germani R (1994) Tecnologia de farinhas mistas: uso de farinha mista de trigo e mandioca na produção de pães. v.1. Brasília, EMBRAPA SPI. 8p.

Fuchs RHB (2005) “Iogurte” de soja suplementado com oligofrutose e inulina. Ciências e Tecnologia de Alimentos, 25:175-181.

Hasler CM (1998) Functional Foods: Their Role in Disease Prevention and Health Promotion. Food Technology, 52:63-70.

Kinouchi FL, Cardello HMAB, Rossi EA \& Telarolli Júnior R (2002) Aceitação do "Iogurte” de Soja Entre Adolescentes. Revista Alimento e Nutrição, 13:131-142.

Minim VPR (2006) Análise sensorial: Estudos com consumidores. Viçosa, Ed. UFV. 225p.

Monteiro MRP, Costa NMB, Oliveira MGA, Pires CV \& Moreira MA (2004) Qualidade protéica de linhagens de soja com ausência do Inibidor de Tripsina Kunitz e das isoenzimas Lipoxigenases Revista de Nutrição, 17:195-205.

Morais AAC \& Silva AL (2000) Valor nutritivo e funcional da soja. Revista Brasileira de Nutrição Clinica, 15:306-315.

Morais AAC, Silva AL \& Damásio ABF (2000) Otimização do uso da soja, Revista Brasileira de Nutrição Clinica, 15:350-357.

Penha LAO, Fonseca ICB, Mandarino JM \& Benassi VT (2007) A soja como alimento: valor nutricional, benefícios para a saúde e cultivo orgânico. B.CEPPA, 25:91-102.

Tsukamoto C, Kudou S, Kikuchi A, Carrão-Panizzi MC, ONO T \& Kitamura-Okubo KK (2001) Isoflavones in soybean products: composition, concentration and physiological effects. In: I Simpósio Brasileiro sobre os Benefícios da Soja para a Saúde Humana, Londrina. Anais, Embrapa Soja. p.9-14. 